Document downloaded from:

http://hdl.handle.net/10251/47181

This paper must be cited as:

Galdames, O.; Sánchez Pérez, EA. (2013). Factorizing Kernel Operators. Integral Equations and Operator Theory. 75(1):13-29. doi:10.1007/s00020-012-2019-z.

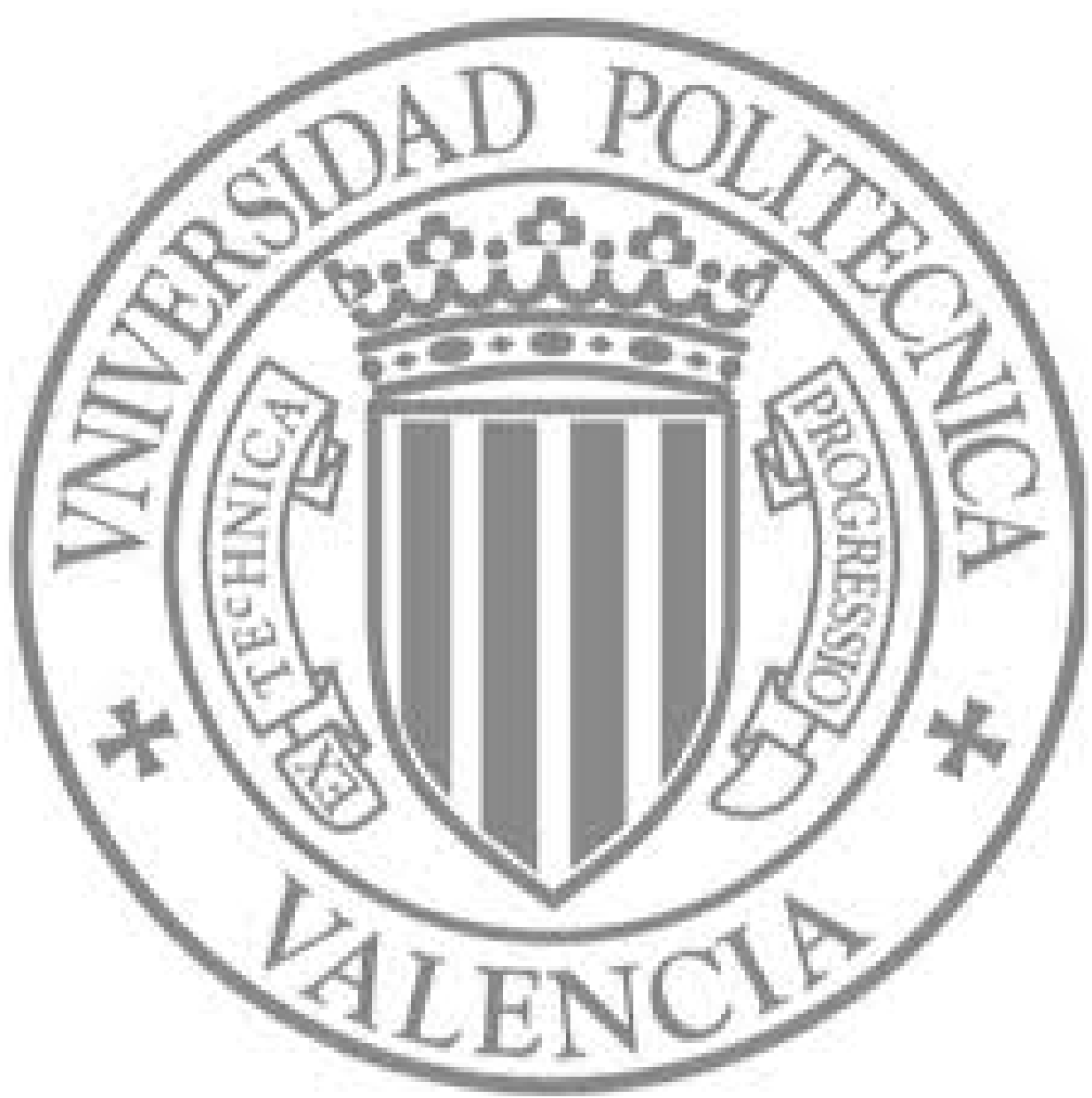

The final publication is available at

http://dx.doi.org/10.1007/s00020-012-2019-z

Copyright Springer Verlag (Germany) 


\title{
Factorizing kernel operators
}

\author{
O. Galdames Bravo and E. A. Sánchez Pérez
}

\begin{abstract}
Consider an operator $T: X(\mu) \rightarrow Y(\mu)$ between Banach function spaces having adequate order continuity and Fatou properties. Assume that $T$ can be factorized through a Banach space as $T=R \circ S$, where $R$ and the adjoint of $S$ are $p$-th power and $q$-th power factorable, respectively. Then a canonical factorization scheme can be given for $T$. We show that it provides a tool for analyzing $T$ that becomes specially useful for the case of kernel operators. In particular, we show that this square factorization scheme for $T$ is equivalent to some inequalities for the bilinear form defined by $T$. Kernel operators are studied from this point of view.
\end{abstract}

Primary 46E30, Secondary 47B38, 46B42, 46B28

Banach function spaces, Köthe duality, $p$-th power factorable operators, factorization, kernel operators.

\section{Introduction}

The class of $p$-th power factorable operators has shown to be a convenient tool for analyzing certain factorization properties of operators between Banach function spaces (see Chapter 5 in [9]). For instance, relevant operators coming from the Fourier analysis as convolution operators and the Fourier transform are examples of such kind of linear maps (see Chapter 7 in [9]). Essentially, this class is defined by an extension property that allows to factorize an operator $T: X(\mu) \rightarrow Y(\mu)$ between two Banach function spaces through the $p$-th power $X(\mu)_{[p]}$ of $X(\mu)$. Interesting properties of linear maps satisfying such requirement can be proved by means of this factorization scheme and its characterizations via spaces of $p$-integrable functions with respect to a vector measure.

In this paper we use this technique to provide some factorization diagrams for kernel operators. In order to do that, it is shown to be necessary to develop the duality theory of $p$-th power factorable operators. We show in Section 2 some basic definitions and some examples and results on $p$-th power factorable kernel operators that can be obtained using the duality formula for $p$-th powers of Banach function spaces. Section 3 is devoted to present our main factorization theorem for operators satisfying some $p$-th power and $q$-th power factorability properties for $T$ and for its adjoint operator $T^{*}$, respectively.

Support of the Ministerio de Ciencia e Innovación under project \#MTM2009-14483-C02-02 (Spain) is gratefully acknowledged. 
This result (Theorem 3.7) involves also the spaces of $p$-integrable functions with respect to a vector measure. Some examples and applications are also given. Finally, in Section 4 we provide the factorization schemes for kernel operators that can be obtained using this technique.

\section{Preliminaries and basic results}

Let $(\Omega, \Sigma, \mu)$ be a finite measure space. If $p$ is a positive extended real number, we write $p^{\prime}$ for the real number satisfying $1 / p+1 / p^{\prime}=1$. A Banach function space $X(\mu)$ over $\mu$ (B.f.s. for short) is an ideal of the space of (equivalence classes of) measurable functions $L^{0}(\mu)$ endowed with a complete norm that is compatible with the $\mu$-a.e. order and such that $L^{\infty}(\mu) \subseteq X(\mu) \subseteq L^{1}(\mu)$ (see p.28 in [8]). For the sake of simplicity we will write $X$ instead of $X(\mu)$ if the measure is clear in the context. Let $m: \Sigma \rightarrow E$ be a countably additive vector measure, where $E$ is a Banach space. For an element $x^{\prime}$ of the dual space $E^{*}$ of $E$, we define the scalar measure $\left\langle m, x^{\prime}\right\rangle$ by $\left\langle m, x^{\prime}\right\rangle(A):=\left\langle m(A), x^{\prime}\right\rangle, A \in \Sigma$. We write $\left|\left\langle m, x^{\prime}\right\rangle\right|$ for the variation of the measure $\left\langle m, x^{\prime}\right\rangle$, and $\|m\|$ for the semivariation of the vector measure $m$. A measure as $\left|\left\langle m, x^{\prime}\right\rangle\right|$ that is equivalent to $m$-in the sense that has the same null sets - is called a Rybakov measure for $m$. Such a measure always exists; we refer to [5] for this notion, and for definitions and basic results on vector measures.

We say that a measurable function $f$ is $m$-integrable if $f \in L^{1}\left(\left|\left\langle m, x^{\prime}\right\rangle\right|\right)$ for all $x^{\prime} \in E^{*}$ and for all $A \in \Sigma$ there exists a unique vector $x_{0} \in E$ such that $\left\langle x_{0}, x^{\prime}\right\rangle=$ $\int_{A} f d\left\langle m, x^{\prime}\right\rangle$ for all $x^{\prime} \in E^{*}$; in this case the notation $\int_{A} f d m:=x_{0}$ is used. Let $\lambda$ be a Rybakov measure for $m$. The space of (equivalence classes of) $m$-integrable functions is denoted by $L^{1}(m)$, which is a Banach function space over $\lambda$ with the norm

$$
\|f\|_{L^{1}(m)}:=\sup _{x^{\prime} \in B_{E^{*}}} \int|f| d\left|\left\langle m, x^{\prime}\right\rangle\right|, \quad f \in L^{1}(m) .
$$

The integration map $I_{m}: L^{p}(m) \rightarrow E$ given by $f \rightsquigarrow \int_{\Omega} f d m$ is always well defined and continuous.

A Banach function space $X(\mu)$ is order continuous if for every sequence $\left(f_{n}\right)_{n} \subset$ $X(\mu), f_{n} \geq 0$, such that $f_{n} \downarrow 0$ we have that $\left\|f_{n}\right\| \downarrow 0$. X( $\mu$ ) has the Fatou property if for every increasing sequence $\left(f_{n}\right)_{n} \subset X(\mu)$ such that $f_{n} \uparrow f$ and $\sup _{n}\left\|f_{n}\right\|<\infty$, then $f \in X(\mu)$ and $\lim _{n}\left\|f_{n}\right\|=\|f\|$. The Banach space of all integral functionals on $X(\mu)$ is the Köthe dual space and is denoted by $X(\mu)^{\prime}$. The topological dual is denoted by $X(\mu)^{*}$. In [8, p.29-30] we can find the following characterizations: $X(\mu)$ is order continuous if and only if $X(\mu)^{\prime}=X(\mu)^{*}$ and $X(\mu)$ has the Fatou property if and only if $X(\mu)^{\prime \prime}=X(\mu)$.

If $0<p<\infty$ and $f \in L^{0}(\mu)$, we will use the notation $f^{p}$ for the function $f^{p}(w):=$ $|f(w)|^{p} \operatorname{sign}\{f(w)\}, w \in \Omega$. The $p$-th power space of a B.f.s. $\left(X(\mu),\|\cdot\|_{X(\mu)}\right)$ is the space

$$
X(\mu)_{[p]}:=\left\{f \in L^{0}(\mu):|f|^{1 / p} \in X(\mu)\right\}
$$

which is a quasi-Banach function space with quasi-norm

$$
\|f\|_{X(\mu)_{[p]}}:=\left\||f|^{1 / p}\right\|_{X(\mu)}^{p} .
$$

This quasi-norm is equivalent to a norm if and only if $X(\mu)$ is $q$-convex (see Proposition 2.23 in [9] and [9, Ch.2] for definitions and basic properties of Banach function spaces). 
Recall that a Banach function space $X(\mu)$ is $q$-convex if there is a constant $K_{q}$ such that for every finite set $f_{1}, \ldots, f_{n} \in X(\mu)$, the inequality

$$
\left\|\left(\sum_{i=1}^{n}\left|f_{i}\right|^{q}\right)^{1 / q}\right\| \leq K_{q}\left(\sum_{i=1}^{n}\left\|f_{i}\right\|^{q}\right)^{1 / q}
$$

holds. It is $q$-concave if there is a constant $K^{q}$ such that the converse inequality

$$
\left(\sum_{i=1}^{n}\left\|f_{i}\right\|^{q}\right)^{1 / q} \leq K^{q}\left\|\left(\sum_{i=1}^{n}\left|f_{i}\right|^{q}\right)^{1 / q}\right\|
$$

holds. If $\mu$ is a finite measure then for every $1 \leq p, X \subseteq X_{[p]}$. Throughout the paper $i_{[p]}$ will denote this inclusion, that will be written as $j_{[q]}$ sometimes for the aim of clarity when $p$-th power and $q$-th power factorable operators are be involved.

A particular case of $p$-th power spaces $(1 \leq p<\infty)$ are the spaces $L^{p}(m)$ of $p$ integrable functions with respect to the vector measure $m$, that are defined by $L^{p}(m):=$ $\left(L^{1}(m)\right)_{[1 / p]}$. In this case, the $p$-th power quasi-norm gives in fact a norm, and $L^{p}(m) \subseteq$ $L^{1}(m)$. The (restricted) integration map will be denoted in this case by $I_{m}^{(p)}: L^{p}(m) \rightarrow E$.

The next basic properties will be used in the paper. More information can be found in Lemma 2.20, Lemma 2.21 and Proposition 2.23 in [9].

Lemma 2.1. Let $X(\mu)$ and $Y(\mu)$ be a couple of Banach function spaces. Then:

1. For all $0<p, r<\infty$ we have that $\left(X(\mu)_{[p]}\right)_{[r]}=X(\mu)_{[p r]}$.

2. $X(\mu) \subseteq Y(\mu)$ if and only if $X(\mu)_{[p]} \subseteq Y(\mu)_{[p]}$ for some $p \in(0, \infty)$.

3. $X(\mu)$ is order continuous if and only if $X(\mu)_{[p]}$ is o.c. for all $p \in(0, \infty)$.

4. If $0<p \leq q<\infty$, then $X(\mu)_{[p]} \subseteq X(\mu)_{[q]}$.

The natural definition of operators on Banach function spaces involving $p$-th powers is the following.

Definition 2.2. Let $1 \leq p<\infty, X(\mu)$ an order continuous Banach function space, and $E$ a Banach space. We say that a linear and continuous operator $T: X(\mu) \rightarrow E$ is $p$-th power factorable if there exists a linear and continuous operator $T_{[p]}: X(\mu)_{[p]} \rightarrow E$, which equals $T$ on $X(\mu) \subseteq X(\mu)_{[p]}$. In other words, the following diagram commutes

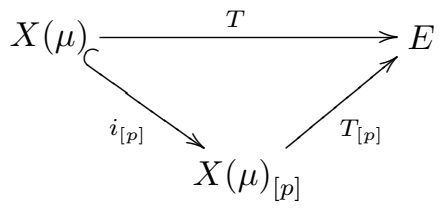

where $i_{[p]}$ is the continuous inclusion.

According to this factorization scheme, notice that for analyzing adjoint operators some information on the dual of the space $X(\mu)_{[p]}$ is required. Recall that for two Banach function spaces $X(\mu)$ and $Y(\mu)$ the space of multipliers from $X(\mu)$ to $Y(\mu)$ is defined as the space

$$
X(\mu)^{Y(\mu)}:=\left\{g \in L^{0}(\mu): g X(\mu) \subseteq Y(\mu)\right\}
$$


If it is nontrivial, the operator norm provides a Banach function space structure for $X(\mu)^{Y(\mu)}$ when the $\mu$-almost everywhere order is considered. Notice that in particular the Köthe dual of $X(\mu)$ is defined as

$$
X(\mu)^{\prime}=\left\{g \in L^{0}(\mu): g X(\mu) \subseteq L^{1}(\mu)\right\},
$$

and so $X(\mu)^{\prime}=X(\mu)^{L^{1}(\mu)}$.

Let us show now a representation formula for the dual of the space $X_{[p]}$, which is naturally related to the space $X(\mu)^{L^{p}(\mu)}$.

Remark 2.3. First, it is important to remark that for an index $p \in(0, \infty), p \neq 1$, we have in general that $\left(X(\mu)^{\prime}\right)_{[p]} \neq\left(X(\mu)_{[p]}\right)^{\prime}$. To see this, assume that $\mu$ is a purely non atomic finite measure. If $\left(X(\mu)^{\prime}\right)_{[p]}=\left(X(\mu)_{[p]}\right)^{\prime}$, then $X(\mu)^{\prime} \subseteq\left(X(\mu)^{\prime}\right)_{[p]}=\left(X(\mu)_{[p]}\right)^{\prime} \subseteq$ $X(\mu)^{\prime}$, and due to Proposition 2.26 in $[9], X(\mu)^{\prime}=\left(X(\mu)^{\prime}\right)_{[p]}=L^{\infty}(\mu)$, which is true if $X(\mu)=L^{1}(\mu)$. For $p>1$ this would imply that $X(\mu)^{\prime}=\left(X(\mu)_{[p]}\right)^{\prime}=\left(L^{1 / p}(\mu)\right)^{\prime}=\{0\}$ (see [13, Ch.15]). If $p<1$, we have that

$$
L^{\infty}(\mu)=L^{\infty}(\mu)_{[p]}=\left(X(\mu)^{\prime}\right)_{[p]}=\left(X(\mu)_{[p]}\right)^{\prime}=L^{(1 / p)^{\prime}}(\mu)=L^{1 / 1-p}(\mu),
$$

and so $p=1$, which is a contradiction.

The main duality identification involving $X_{[p]}$ and $X^{L^{p}}$ is

$$
\left(X(\mu)_{[p]}\right)^{\prime}=\left(X(\mu)^{L^{p}(\mu)}\right)_{[p]},
$$

that holds for all $p \in(0, \infty)$ (see Proposition 2.29(ii),(iv) in [9]).

We finish this section with two results on sufficient conditions for the factorization of kernel operators through $p$-th power spaces. Let us introduce some definitions and notations on kernel operators. We say that a function $K: \Omega \times \Omega \rightarrow \mathbb{R}$ that is integrable with respect to $\mu$ in each coordinate is a kernel function. Consider a couple of Banach function spaces $X(\mu)$ and $Y(\mu)$, and assume that the formula

$$
T_{K}(f)(x):=\int K(x, y) f(y) d \mu(y)
$$

defines a continuous map $T_{K}: X(\mu) \rightarrow Y(\mu)$. Then we say that $T_{K}$ is a kernel operator.

We denote by $\|K\|_{X(\mu) y}=\|K\|_{X(\mu) y}(x)$ to the function $x \rightsquigarrow\|K(x, \cdot)\|_{X(\mu)} ;\|K\|_{X(\mu) x}$ is defined in the same way for the second coordinate. Note that a necessary condition for this to be well defined is that for all $x \in \Omega$ the corresponding function $y \rightsquigarrow K(x, y)$ belongs to $X(\mu)$. In what follows this requirement is implicitly assumed when the functions $\|K\|_{X(\mu) y}$ and $\|K\|_{X(\mu) x}$ are considered. For the aim of simplicity, when $X(\mu)=L^{q}(\mu)$ we will write $\|K\|_{q y}$ and $\|K\|_{q x}$.

Proposition 2.4. Let $X(\mu)$ be a Banach function subspace of $L^{p}(\mu)$, and $Y(\mu)$ be a $p$ convex Banach function space with the Fatou property. Let $K$ be a kernel function satisfying that there is $q \geq p$ such that \|\|$K\left\|_{Y(\mu)_{[q]} x}\right\|_{p^{\prime}}<\infty$. Then the kernel operator $T_{K}: X(\mu) \rightarrow Y(\mu)$ defined by $K$ can be extended to the space $L^{p}(\mu)$. 
Proof. First note that $\|\cdot\|_{q}$ is $p$-concave, since $q \geq p$. Then we can use the generalized Minkowski inequality (see [12, Theorem 2.3]) and the integral Hölder inequality. Let $f \in X(\mu)$. We have that

$$
\begin{aligned}
\left\|T_{K}(f)\right\|_{Y(\mu)} & =\left\|\int_{\Omega} K(x, y) f(y) d \mu(y)\right\|_{Y(\mu)} \\
& \leq\|\||K(x, y)|^{1 / q}|f(y)|^{1 / q}\left\|_{q y}^{q}\right\|_{Y(\mu)} \\
& \leq M\|\||K(x, y)|^{1 / q}|f(y)|^{1 / q}\left\|_{Y(\mu) x}\right\|_{q y}^{q} \\
& =M \int_{\Omega}\left(\left\||K|^{1 / q}\right\|_{Y(\mu) x}^{q}\right)(y)|f(y)| d \mu(y) \\
& \leq M\|\| K\left\|_{Y(\mu)_{[q]} x}\right\|_{p^{\prime}}\|f\|_{p}
\end{aligned}
$$

Therefore, as a consequence of the inclusion $X(\mu) \subseteq L^{p}(\mu)$ and the facts that simple functions are in $X(\mu)$ and are dense in $L^{p}(\mu), T_{K}$ can be extended continuously to the space $L^{p}(\mu)$.

Proposition 2.5. Let $r, s, p \geq 1$ be such that $\frac{1}{r}=\frac{1}{p}+\frac{1}{s}$ and let $K$ be a kernel function such that \|\|$K\left\|_{q x}\right\|_{s / r}<\infty$. Then the kernel operator associated to $K, T_{K}: L^{p}(\mu) \rightarrow L^{q}(\mu)$ is $r$-th power factorable.

Proof. Since $r \leq p, L^{p}(\mu) \subseteq L^{r}(\mu)$. Let $f$ be a simple function. Then, using Minkowski's inequality and Hölder's inequality we obtain

$$
\begin{aligned}
\left(\left\|T_{K}\left(f^{r}\right)\right\|_{q}\right)^{1 / r} & =\left(\int_{\Omega}\left|\int_{\Omega} K(x, y) f(y)^{r} d \mu(y)\right|^{q} d \mu(x)\right)^{1 /(q r)} \\
& \leq\left[\int_{\Omega}\left(\int_{\Omega}\left|K(x, y) f(y)^{r}\right|^{q} d \mu(x)\right)^{1 / q} d \mu(y)\right]^{1 / r} \\
& =\left[\int_{\Omega}|f(y)|^{r}\left(\int_{\Omega}|K(x, y)|^{q} d \mu(x)\right)^{1 / q} d \mu(y)\right]^{1 / r} \\
& =\left(\int_{\Omega}|f(y)|^{r}\|K\|_{q x}(y) d \mu(y)\right)^{1 / r} \\
& \leq\left(\int_{\Omega}|f(y)|^{p} d \mu(y)\right)^{1 / p}\left(\int_{\Omega}\|K\|_{q x}^{s / r}(y) d \mu(y)\right)^{1 / s} \\
& =\left\|\left(\|K\|_{q x}\right)^{1 / r}\right\|_{s}\left(\int_{\Omega}|f(y)|^{p} d \mu(y)\right)^{1 / p} \\
& =\left\|\left(\|K\|_{q x}\right)^{1 / r}\right\|_{s}\|f\|_{p} .
\end{aligned}
$$

Using that simple functions are dense in $L^{p}(\mu)$, these inequalities proves that there is a constant $C$ such that $\left\|T_{K}(g)\right\|_{q} \leq C\left\||g|^{1 / r}\right\|_{p}^{r}=C\|g\|_{L^{p}(\mu)_{[r]}}$ for all $g \in L^{p}(\mu)$. Thus we have that $T_{K}: L^{p}(\mu) \rightarrow L^{q}(\mu)$ is $r$-th power factorable. 
Notice that these results work also for the adjoint operator $T_{K}^{\prime}$, since it is again a kernel operator with a similar kernel.

Example 2.6. The Volterra operator $V: L^{p}[0,1] \rightarrow L^{q}[0,1]$ has kernel function $K(x, y):=$ $\chi_{[0, x]}(y)$. Hence, for $r, s>1$ such that $\frac{1}{r}=\frac{1}{p}+\frac{1}{s}$ we have that \|\|$K\left\|_{q_{x}}\right\|_{s / r}=\left(\frac{q r}{q r+s}\right)^{r / s}$ and then $V$ is $r$-th power factorable for $1 \leq r \leq p$ (see Example 5.9 in [9]).

\section{Square factorization diagrams}

A $p$-th power factorable operator can be easily characterized by means of a factorization diagram, as has been shown in Section 2 (see Lemma 5.3 in [9] for the case of a $\mu$ determined operator). In this section we provide a square factorization scheme for the generalization of the notion of $p$-th power factorable operator that is given in the following definition. Through the rest of the paper, we write our results for operators between Banach function spaces over the same finite measure $\mu$ for the sake of clarity. However, the reader can notice that the results work also for Banach function spaces over different finite measures.

Definition 3.1. Let $X(\mu)$ and $Y(\mu)$ be B.f.s.'s. Then we say that an operator $T: X(\mu) \rightarrow$ $Y(\mu)$ is $(p, q)$-th power factorable if there exist a Banach space $E$ and operators $R: X(\mu) \rightarrow$ $E$ and $S: E \rightarrow Y(\mu)$ such that $T=S \circ R, R$ is $p$-th power factorable and the Köthe adjoint operator of $S, S^{\prime}: Y(\mu)^{\prime} \rightarrow E^{*}$ is $q$-th power factorable.

Let us show first a relevant example of this class of operators.

Example 3.2 (Hardy type operators). Let $s \geq 0$ and consider the kernel operator $H_{s}$ with kernel function $K(x, y):=\frac{1}{x^{s}} \chi_{[0, x]}(y)$, i.e.

$$
\left(H_{s} f\right)(x)=\int_{0}^{1} K(x, y) f(y) d y=\int_{0}^{1} \frac{1}{x^{s}} f(y) \chi_{[0, x]}(y) d y=\frac{1}{x^{s}} \int_{0}^{x} f(y) d y .
$$

Note that by Hölder's inequality the operator $H_{s}: L^{u}[0,1] \rightarrow L^{v}[0,1]$ is always well defined and continuous for $1 \leq v<u$ when $s<1 / v-1 / u$ (in fact, it is continuous in more cases, see for instance [2, Theorem 3.10]). Under these restrictions for $u, v$ and $s$ we can consider the following factorization. For $f(x)=x^{-s}$ and the Volterra operator $V: L^{u}[0,1] \rightarrow L^{u}[0,1]$, we can write

$$
H_{s}=M_{f} \circ V: L^{u}[0,1] \stackrel{V}{\longrightarrow} L^{u}[0,1] \stackrel{M_{f}}{\longrightarrow} L^{v}[0,1] .
$$

It is known that $V$ is $p$-th power factorable for all $1 \leq p \leq u$ (see [9, Example 5.9]). On the other hand, notice that $\left(M_{f}\right)^{\prime}=M_{f}: L^{v^{\prime}}[0,1] \rightarrow L^{u^{\prime}}[0,1]$ and for $g \in L^{u}[0,1]$ we have that $M_{f}(g) \in L^{v}[0,1]$. Take then an index $1<t \leq v^{\prime}$ such that $s<1 / u^{\prime}-t / v^{\prime}$ (note that these requirements are compatible with the restrictions on the indexes written above). Then a direct computation usinng Hölder's inequality gives the continuity of the map $M_{f}: L^{v^{\prime} / t} \rightarrow L^{u^{\prime}}$, i.e. $\left(M_{f}\right)^{\prime}$ is $t$-th power factorable. Consequently, $H_{s}$ is $(p, t)$-th factorable for all $1 \leq p \leq u$. 
In Chapters 4 and 5 of [9] it is studied how an operator can be extended to the space of $p$-integrable functions with respect to a vector measure in the following way. If $X(\mu)$ is an order continuous Banach function space and $T: X \rightarrow E$ is an operator, we know that $m_{T}(A):=T\left(\chi_{A}\right)$ is a vector measure. For the case of $p$-th power factorable operators, if $m_{T}$ has the same null sets that $\mu$ (i.e. $T$ is $\mu$-determined), we have that $X(\mu)$ is included into $L^{p}\left(m_{T}\right)$ and so the operator $T$ can be extended to $L^{p}\left(m_{T}\right)$. In this case, the inclusion map $[i]: X \rightarrow L^{p}\left(m_{T}\right)$, given by $f \rightsquigarrow[i](f)=[f]$ is injective (here $[f]$ denotes the equivalence class of $f$ with respect to $\left.\left\|m_{T}\right\|\right)$. However, injectivity is not needed for obtaining a factorization of $T$ through $L^{p}\left(m_{T}\right)$. Without the injectivity assumption, taking into account that 1-th power factorability coincides with continuity, the map $[i]$ is still well defined and continuous, and we always have that $[i](X(\mu)) \subseteq L^{1}\left(m_{T}\right)$ continuously (see [3]). We will denote this relation by $X(\mu) \hookrightarrow_{[i]} L^{1}\left(m_{T}\right)$. In the case that we can assure that in fact $[i](X(\mu))$ is included in the subspace $L^{p}\left(m_{T}\right)$ of $L^{1}\left(m_{T}\right)$ for any $p>1$, we will also write $X(\mu) \hookrightarrow_{[i]} L^{p}\left(m_{T}\right)$.

The following lemma is a generalization of part $(\mathrm{i}) \Leftrightarrow($ iii) in Theorem 5.7 of [9] without the assumption of $T$ being $\mu$-determined. The ideas that prove it can be found in [4].

Lemma 3.3. Let $1 \leq p<\infty$, and let $X(\mu)$ be an order continuous Banach function space. Consider a Banach space $E$. The following assertions for an operator $T: X \rightarrow E$ are equivalent.

1. $T$ is $p$-th power factorable, i.e. there exists $C>0$ such that

$$
\|T f\|_{E} \leq C\|f\|_{X_{[p]}}, \quad f \in X .
$$

2. The map $X \hookrightarrow_{[i]} L^{p}\left(m_{T}\right)$ is well defined (and so continuous).

Proof. Assume that 1 is satisfied. Then the operator $T: X \rightarrow E$ is continuous with the relative topology on $X$ induced by $X_{[p]}$. Since - by the order continuity assumption$X$ is dense in $X_{[p]}$ we know that $T$ can be uniquely extended to $X_{[p]}$. Let us denote by $T_{[p]}: X_{[p]} \rightarrow E$ to this extension. Note that $m_{T_{[p]}}=m_{T}$. By the comments above we can assure that $X_{[p]} \hookrightarrow_{[i]} L^{1}\left(m_{T_{[p]}}\right)=L^{1}\left(m_{T}\right)$. Finally for $f \in X$ we have that $|f|^{p} \in X_{[p]} \hookrightarrow[i] L^{1}\left(m_{T}\right)$, hence

$$
\begin{gathered}
\|[f]\|_{L^{p}\left(m_{T}\right)}=\left\|\left(|f|^{p}\right)^{1 / p}\right\|_{L^{p}\left(m_{T}\right)}=\left\|\left[|f|^{p}\right]\right\|_{L^{1}\left(m_{T}\right)}^{1 / p} \\
\leq K^{1 / p}\left\||f|^{p}\right\|_{X_{[p]}^{1 / p}}^{1 / p}=K^{1 / p}\|f\|_{X} .
\end{gathered}
$$

So we have that $X \hookrightarrow_{[i]} L^{p}\left(m_{T}\right)$.

For the converse suppose that $X \hookrightarrow_{[i]} L^{p}\left(m_{T}\right)$. Then the calculation above shows that $X_{[p]}{ }_{[i]} L^{1}\left(m_{T}\right)$. In consequence, we have that $T=I_{m_{T}} \circ[i] \circ i_{[p]}$. Thus, for $f \in X$, we have that

$$
\|T f\|_{E}=\left\|\left(I_{m_{T}} \circ[i] \circ i_{[p]}\right) f\right\|_{E} \leq\left\|I_{m_{T}} \circ[i]\right\|\|f\|_{X_{[p]}} .
$$

This finishes the proof. 
Remark 3.4. Note that the assumption on the $p$-th power factorability of a non trivial operator $T$ together with the order continuity of $X$ implies that $\left(X_{[p]}\right)^{*}$ is nontrivial. Certainly, the composition of $T$ with a functional $x^{*} \in E^{*}$ produces a continuous functional $z^{*}$ on the quasi-normed space $\left(X,\|\cdot\|_{X_{[p]}}\right)$ that together with the density of $X$ in $X_{[p]}$ and the order continuity gives that $z^{*} \in\left(X_{[p]}\right)^{*}$.

We will call the map $[i]$ — that depends on $\mu$ and $m_{T}$ - an inclusion/quotient map. Notice that the Köthe adjoint map $[i]^{\prime}$ is injective, since $\left\|m_{T}\right\|$ is always absolutely continuous with respect to $\mu$. This general point of view for the understanding of the integral extension of operators can be found in [4], without assuming $T$ to be $\mu$-determined. Also notice that the map $[i]^{\prime}$ depends on the Rybakov measure $\nu$ for $m_{T}$ that is considered for defining $L^{p}\left(m_{T}\right)$ as a Banach function space over $\nu$. In fact, in order to simplify the notation, we will fix a Rybakov measure $\nu$ for defining the map $[i]^{\prime}$. In general, the injective map $[i]^{\prime}$ do not define a natural inclusion, i.e. $[i]^{\prime}(f)$ is not necessarily equal to $[f]$. Let $d \nu / d \mu$ be the Radon-Nikodým derivative of $\nu$ with respect to $\mu$. Then the duality relation is given by

$$
\left\langle f,[i]^{\prime}(g)\right\rangle=\int f[i]^{\prime}(g) d \mu=\int[i](f) g d \nu=\int[i](f) g\left(\frac{d \nu}{d \mu}\right) d \mu,
$$

-where $f \in X(\mu)$ and $g \in\left(L^{p}\left(m_{T}\right)\right)^{\prime}$ — that do not produce a proper inclusion map, but it is the key for finding the optimal range of an operator with values in a Banach function space, in the sense that is explained in [7]. Observe that if $[i]$ is injective (that is, $\mu$ is equivalent to $\left.\left\|m_{T}\right\|\right)$, then $[i]^{\prime}(g)=(d \nu / d \mu) \cdot g$, in other words $[i]^{\prime}$ is given by a multiplication by a positive function.

The following result provides the main characterization for $(p, q)$-th power factorable operators.

Theorem 3.5. Let $X(\mu)$ and $Y(\mu)$ be order continuous Banach function spaces with the Fatou property such that $Y(\mu)^{\prime}$ is also order continuous. Let $T: X(\mu) \rightarrow Y(\mu)$ be an operator. Then there exists an operator

$$
T_{[p, q]}: X(\mu)_{[p]} \rightarrow\left(Y(\mu)_{[q]}^{\prime}\right)^{\prime}
$$

such that $T=\left(i_{[q]}\right)^{\prime} \circ T_{[p, q]} \circ i_{[p]}$ if and only if $T$ is $(p, q)$-th power factorable.

Proof. Assume first that we have an operator $T_{[p, q]}: X(\mu)_{[p]} \rightarrow\left(\left(Y(\mu)^{\prime}\right)_{[q]}\right)^{\prime}$ such that $T=\left(i_{[q]}\right)^{\prime} \circ T_{[p, q]} \circ i_{[p]}$ (it is implicitly assumed that the inclusions $i_{[p]}$ and $i_{[q]}$ are welldefined). Then $T_{[p, q]} \circ i_{[p]}$ is - by definition - $p$-th power factorable. The order continuity of $Y^{\prime}$ implies the order continuity of the Banach function space $\left(Y^{\prime}\right)_{[q]}$, and so $\left(\left(Y^{\prime}\right)_{[q]}\right)^{*}=$ $\left(\left(Y^{\prime}\right)_{[q]}\right)^{\prime}$ (see Remark 2.6 in [9]). Consequently, $Y^{\prime}=Y^{*}$ and $\left(Y^{\prime}\right)_{[q]} \subseteq\left(\left(\left(Y^{\prime}\right)_{[q]}\right)^{\prime}\right)^{*}$. Therefore, $\left(\left(i_{[q]}\right)^{\prime}\right)^{*}: Y^{\prime}=Y^{*} \rightarrow\left(\left(Y(\mu)^{\prime}{ }_{[q]}\right)^{\prime}\right)^{*}$ factorizes through the inclusion map $Y^{\prime} \hookrightarrow\left(Y^{\prime}\right)_{[q]}$, and so it is $q$-th power factorable.

For the converse, suppose that $T$ is $(p, q)$-th power factorable and consider the Banach space $E$, the $p$-th power factorable operator $R: X(\mu) \rightarrow E$ and the operator $S: E \rightarrow Y(\mu)$ such that $S^{\prime}$ is $q$-th power factorable and $T=S \circ R$. Then by [9, Lemma 
5.3] we have an extension as

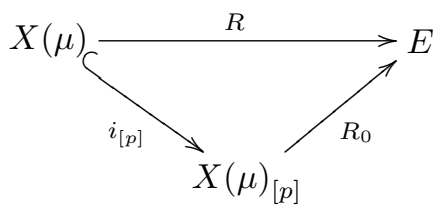

On the other hand, by the same result we have a factorization for $S^{\prime}$ as

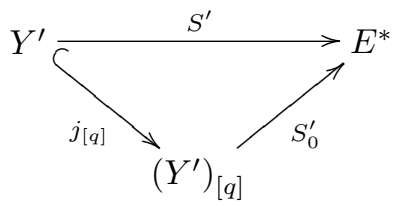

By dualizing and taking into account the properties of the spaces involved, we can obtain the following factorization scheme.

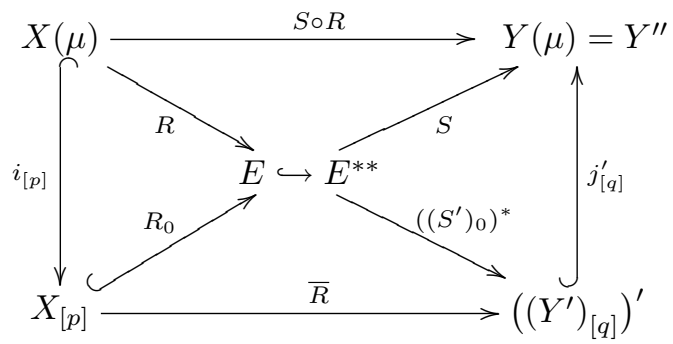

where $\bar{R}=\left(\left(S^{\prime}\right)_{0}\right)^{*} \circ R_{0}$ plays the role of $T_{[p, q]}$. Since $\left(i_{[q]}\right)^{\prime \prime}=i_{[q]}$, we obtain the result.

Example 3.6. Classical factorization theorems provide easy examples of factorizations as the one above for the case $(1,1)$. Suppose that we have a weakly compact operator $T: X(\mu) \rightarrow Y(\mu)$, where $X(\mu)$ and $Y^{\prime}(\mu)$ are order continuous. Since each weakly compact operator factorizes through a reflexive Banach space (see for instance [5, Corollary VIII.4.9]), we can find a factorization for $T$ as

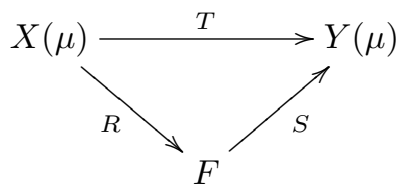

where $F$ is a reflexive Banach space. In particular, $R$ can be extended to its optimal domain $L^{1}\left(m_{R}\right)$ and the same holds for $S^{\prime}:(Y(\mu))^{\prime} \rightarrow F^{*}$ to the corresponding space $L^{1}\left(m_{S^{\prime}}\right)$. Consequently, $T$ is $(1,1)$-th factorable, and the factorization space is reflexive.

The following theorem provides a factorization for $(p, q)$-th power factorable operators involving spaces $L^{p}(m)$ for a vector measure $m$. Roughly speaking they are the class of operators that allows factorization schemes through an operator from $L^{p}\left(m_{1}\right)$ to 
$\left(L^{q}\left(m_{2}\right)\right)^{\prime}$. In a sense, they can be considered as a generalization of the following situation without the strong geometric requirements on $T$ that are necessary in this case. Let $1 \leq q, p<\infty$. Consider a $p$-convex and $q$-concave operator $T: X(\mu) \rightarrow Y(\mu)$ (see $[8$, Chapter 1.d]). If $\theta \in(0,1)$, let us define $p_{\theta}:=\frac{p}{\theta+(1-\theta) p}$ and $q_{\theta}:=\frac{q}{1-\theta}$. By [10, Theorem 15] we know that it is possible to find a factorization of $T$ as $T=S \circ R$ where $S$ is $p_{\theta}$-convex and $R$ is $q_{\theta}$-concave for all $\theta \in(0,1)$. Moreover, by [11, Sec. 2, Corollary 7] there is a Banach lattice $E$ such that it is $p_{\theta}$-concave and $q_{\theta}$-convex, and $T$ factorizes through $E$. We can apply now Maurey's Theorem [1, Theorem 7.1.2] to factorize $T$ as

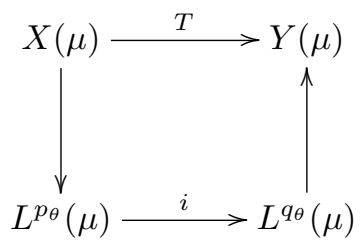

The next result shows that $(p, q)$-th power factorable operators can be considered as a vector measure version of such kind of factorization scheme (see (4) and (5) in the theorem below).

Theorem 3.7. Let $X(\mu)$ be an order continuous B.f.s., and $Y(\mu)$ be Fatou and order continuous B.f.s. such that $Y(\mu)^{\prime}$ is order continuous. Let $T: X(\mu) \rightarrow Y(\mu)$ be an operator. Then the following statements are equivalent:

1. $T$ is $(p, q)$-th power factorable.

2. There exists some $K>0$ such that

$$
|\langle T(f), g\rangle| \leq K\|f\|_{X(\mu)_{[p]}}\|g\|_{\left(Y(\mu)^{\prime}\right)_{[q]}}, \quad f \in X(\mu), g \in Y(\mu)^{\prime},
$$

3. There exists an operator

$$
T_{[p, q]}: X(\mu)_{[p]} \rightarrow\left(Y(\mu)^{\prime}{ }_{[q]}\right)^{\prime}
$$

such that the following diagram is commutative

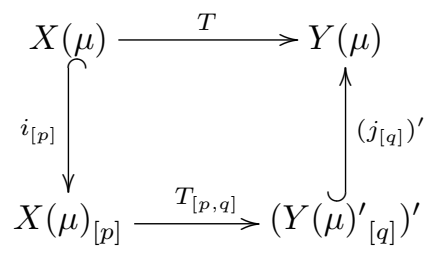

4. There exist two operators $F: X \rightarrow\left(\left(Y^{\prime}\right)_{[q]}\right)^{\prime}$ and $G: Y^{\prime} \rightarrow\left(Y^{\prime}\right)_{[q]}$ and an operator $H: L^{p}\left(m_{F}\right) \rightarrow\left(L^{q}\left(m_{G^{\prime}}\right)\right)^{\prime}$ such that the following diagram is commutative

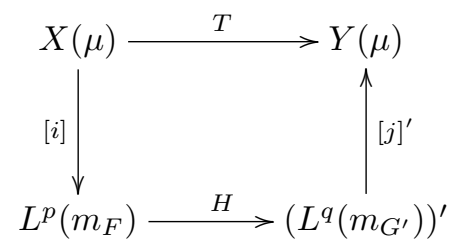


5. There exist an operator $H$ and a Banach space $E$ and two operators $R: X \rightarrow E$ and $S: E \rightarrow Y$ such that $T=S \circ R$ and that the following diagram is commutative

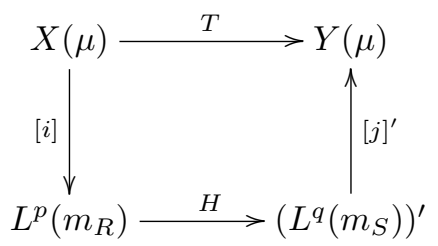

Proof. $1 \Leftrightarrow 3$ was proved in Theorem 3.5. In order to achieve $2 \Rightarrow 3$ we will show that the operator $T$ is $\left(\|\cdot\|_{\left(Y^{\prime}\right)_{[q]}}-\|\cdot\|_{X_{[p]}}\right)$-continuous. Let $s$ be a simple function. By hypothesis we have that $|\langle T(f), s\rangle| \leq K\|f\|_{X_{[p]}}\|s\|_{\left(Y^{\prime}\right)_{[q]}}$, for all $f \in X$. In consequence, for all simple functions $s \in B_{\left(Y^{\prime}\right)_{[q]}}$ we have that $|\langle T(f), s\rangle| \leq K\|f\|_{X_{[p]}}$, i.e.

$$
\sup _{s}|\langle T(f), s\rangle| \leq K\|f\|_{X_{[p]}},
$$

where the supremum is taken over all the simple functions $s$ in the ball $B_{\left(Y^{\prime}\right)_{[q]}}$. The density of the simple functions in $\left(Y^{\prime}\right)_{[q]}$ (recall that $Y^{\prime}$ is order continuous), and the continuity of $T$ allows us to extend this inequality to all $g \in B_{\left(Y^{\prime}\right)_{[q]}}$ in order to obtain

$$
\|T f\|_{\left(\left(Y^{\prime}\right)_{[q]}\right)^{\prime}}=\sup _{g \in B_{\left(Y^{\prime}\right)[q]}}|\langle T(f), g\rangle| \leq K\|f\|_{X_{[p]}} .
$$

Then we can extend $T$ to $X_{[p]}$ ensuring moreover that the range of $T$ must be in $\left(\left(Y^{\prime}\right)_{[q]}\right)^{\prime}$. In other words, there exists $T_{[p, q]}: X_{[p]} \rightarrow\left(\left(Y^{\prime}\right)_{[q]}\right)^{\prime}$ such that $T=j_{[q]}^{\prime} \circ T_{[p, q]} \circ i_{[p]}$ where $i_{[p]}$ is the inclusion of $X$ into $X_{[p]}$ and $j_{[q]}$ the inclusion of $Y^{\prime}$ into $\left(Y^{\prime}\right)_{[q]}$.

$$
\begin{aligned}
& 3 \Rightarrow 2 \text { Let } f \in X(\mu) \text { and } g \in Y(\mu)^{\prime} \text {. Then } \\
& \qquad \begin{aligned}
|\langle T(f), g\rangle| & =\left|\left\langle\left(j_{[q]}\right)^{\prime} \circ T_{[p, q]} \circ i_{[p]}(f), g\right\rangle\right| \\
& =\left|\left\langle T_{[p, q]} \circ i_{[p]}(f),\left(j_{[q]}\right)^{\prime \prime}(g)\right\rangle\right| \\
& \leq\left\|T_{[p, q]}\right\|\left\|i_{[p]}(f)\right\|_{X(\mu)_{[p]}}\left\|j_{[q]}^{\prime \prime}(g)\right\|_{\left(Y(\mu)^{\prime}{ }_{[q]}\right)^{\prime \prime}} \\
& \leq K\|f\|_{X(\mu)_{[p]}}\|g\|_{Y(\mu)^{\prime}{ }_{[q]}},
\end{aligned}
\end{aligned}
$$

where $K=\left\|T_{[p, q]}\right\|\left\|i_{[p]}\right\|\left\|\left(j_{[q]}\right)^{\prime \prime}\right\|$.

$3 \Rightarrow 4$ Let us define $E:=\left(\left(Y(\mu)^{\prime}\right)_{[q]}\right)^{\prime}$. Consider the $p$-th power factorable map $F:=T_{[p, q]} \circ i_{[p]}: X \rightarrow E$ and the $q$-th power factorable map $G:=j_{[q]}$ given by the inclusion of $Y^{\prime}$ into $\left(Y^{\prime}\right)_{[q]}$. Using the order continuity and Fatou properties of the involved spaces and the characterization given in Lemma 3.3 we obtain the factorization

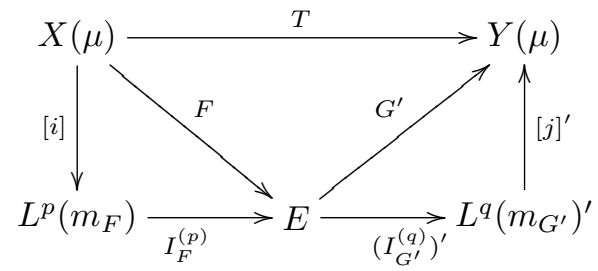


where $H:=\left(I_{G^{\prime}}^{(q)}\right)^{\prime} \circ I_{F}^{(p)}$.

Note that the proof of the previous implication gives in fact also $3 \Rightarrow 5$, for $E=$ $\left(\left(Y(\mu)^{\prime}\right)_{[q]}\right)^{\prime}$ and $S=G^{\prime} .4 \Rightarrow 5$ is obvious. Finally, for $5 \Rightarrow 1$ we have by hypothesis that $X(\mu) \hookrightarrow_{[i]} L^{p}\left(m_{R}\right)$ and that $Y(\mu)^{\prime} \hookrightarrow_{[j]} L^{q}\left(m_{S^{\prime}}\right)$. By Lemma 3.3 we have that $R$ is $p$-th power factorable and $S^{\prime}$ is $q$-th power factorable and thus the result is obtained.

\section{4. $(p, q)$-th power factorable kernel operators}

In this section we apply the results that we have obtained in the previous one in the setting of the kernel operators. Under certain requirements, they will allow us to determine when a kernel that defines an operator between a couple of Banach function spaces defines in fact an operator between $L^{p}$-spaces.

Let us introduce first some notation. As in the rest of the paper, let $(\Omega, \Sigma, \mu)$ be a finite measure space. Consider a function $h: \Omega \rightarrow \mathbb{R}$. Then we can define a function $\Omega \times \Omega \rightarrow \mathbb{R}$ by $h(x, y):=h(x) \chi_{\Omega}(y)$; in this case we will write $h(x)$ for the aim of simplicity. Consider a function $K: \Omega \times \Omega \rightarrow \mathbb{R}^{+}$that is integrable with respect to the product measure $\mu \otimes \mu$. We will call such a function a kernel, and we will denote by $T_{K}$ the associated kernel operator. Clearly, the relation $C \rightsquigarrow \int_{C} K \mu \otimes \mu, C \in \Sigma \otimes \Sigma$ defines a measure in the product space $(\Omega \times \Omega, \Sigma \otimes \Sigma, \mu \otimes \mu)$. In what follows we will call $\eta$ to this measure.

Theorem 4.1. Let $X(\mu)$ and $Y(\mu)$ be Fatou and order continuous Banach function spaces such that $Y(\mu)^{\prime}$ is order continuous. Let $K \in L^{1}(\mu \otimes \mu)$ be an integrable kernel and fix $p, q \geq 1$. Suppose that for all $f \in X(\mu)$ and $g \in Y(\mu)^{\prime}$

$$
\|f(x) g(y)\|_{L^{1}(\eta)} \leq C\|f(x)\|_{L^{p}(\eta)}\|g(y)\|_{L^{q}(\eta)},
$$

for a constant $C>0$. Suppose also that the kernel operator $T_{K}: X(\mu) \rightarrow Y(\mu)$ is $p^{2}$-th power factorable and $T_{K}^{\prime}$ is $q^{2}$-th power factorable. Then $T_{K}$ is $(p, q)$-th power factorable.

Proof. For the aim of clarity we write $d x$ and $d y$ for $d \mu(x)$ and $d \mu(y)$. We can use the Tonelli's Theorem and the fact that $T$ is $p^{2}$-th power factorable to obtain

$$
\begin{aligned}
\int_{\Omega \times \Omega}|f(y)|^{p} d \eta & =\int_{\Omega \times \Omega}|f(y)|^{p} \chi_{\Omega}(x) K(x, y) d x \otimes d y \\
& =\int_{\Omega}\left(\int_{\Omega}|f(y)|^{p} K(x, y) d y\right) \chi_{\Omega}(x) d x \\
& \leq\left\|\chi_{\Omega}\right\|_{Y(\mu)^{\prime}} \sup _{u \in B_{Y(\mu)^{\prime}}} \int_{\Omega}\left(\int_{\Omega}|f(y)|^{p} K(x, y) d y\right) u(x) d x \\
& =\left\|\chi_{\Omega}\right\|_{Y(\mu)^{\prime}} \sup _{u \in B_{Y(\mu)^{\prime}}}\left|\left\langle T\left(|f|^{p}\right), u\right\rangle\right| \\
& =\left\|\chi_{\Omega}\right\|_{Y(\mu)^{\prime}}\left\|T\left(|f|^{p}\right)\right\|_{Y(\mu)} \leq\left\|\chi_{\Omega}\right\|_{Y(\mu)^{\prime}} Q_{1}\left\||f|^{1 / p}\right\|_{X(\mu)}^{p^{2}} .
\end{aligned}
$$

Analogously, by the $q^{2}$-th power factorability of $T^{\prime}$, we have that

$$
\int_{\Omega \times \Omega}|g(x)|^{q} d \eta \leq\left\|\chi_{\Omega}\right\|_{X(\mu)} Q_{2}\left\||g|^{1 / q}\right\|_{Y(\mu)^{\prime}}^{q^{2}} .
$$


In consequence we obtain

$$
\begin{aligned}
|\langle T(f), g\rangle| & =\left|\int_{\Omega}\left(\int_{\Omega} f(x) K(x, y) d x\right) g(y) d y\right|=\left|\int_{\Omega \times \Omega} f(x) g(y) d \eta\right| \\
& \leq\|f g\|_{L^{1}(\eta)} \leq C\|f\|_{L^{p}(\eta)}\|g\|_{L^{q}(\eta)} \leq D\|f\|_{X(\mu)_{[p]}}\|g\|_{\left(Y(\mu)^{\prime}\right)_{[q]}},
\end{aligned}
$$

where $D=C\left\|\chi_{\Omega}\right\|_{X(\mu)}\left\|\chi_{\Omega}\right\|_{Y(\mu)^{\prime}} Q_{1} Q_{2}$. Then we apply Theorem 3.7 in order to obtain that $T$ is $(p, q)$-th power factorable.

Hölder's inequality provides the following result.

Corollary 4.2. Let $X(\mu)$ and $Y(\mu)$ be Fatou and order continuous Banach function spaces such that $Y(\mu)^{\prime}$ is order continuous. Let $K \in L^{1}(\mu \otimes \mu)$ be an integrable kernel and fix $p, q \geq 1$ such that $\frac{1}{p}+\frac{1}{q} \leq 1$. Suppose that the kernel operator $T_{K}: X(\mu) \rightarrow Y(\mu)$ is $p^{2}$-th power factorable and $T_{K}^{\prime}$ is $q^{2}$-th power factorable. Then $T_{K}$ is $(p, q)$-th power factorable.

Corollary 4.3. Under the same hypothesis of Theorem 4.1 and assuming that $1 / p+1 / q=1$ and that $T$ is a positive operator, we have that $T$ is factorable through $L^{p}$-spaces of scalar measures, i.e. the following diagram is commutative

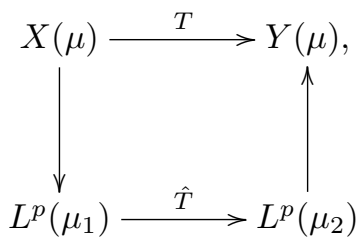

where $\mu_{1}$ and $\mu_{2}$ are positive finite measures.

Proof. By Theorem 4.1 we obtain an operator from $L^{p}\left(m_{R}\right)$ to $\left(L^{q}\left(m_{S^{\prime}}\right)\right)^{\prime}$, where $R$ and $S$ are operators. In fact, the proof of Theorem 4.1 makes clear that $R$ and $S^{\prime}$ can be chosen to be positive. On the other hand, since $T$ is a positive operator we obtain by [8, Proposition 1.d.9],

$$
\left\|\left(\sum_{i=1}^{N}\left|T\left(f_{i}\right)\right|^{p}\right)^{1 / p}\right\|_{\left(L^{q}\left(m_{S^{\prime}}\right)\right)^{\prime}} \leq\left\|\left(\sum_{i=1}^{N}\left|f_{i}\right|^{p}\right)^{1 / p}\right\|_{L^{p}\left(m_{R}\right)}
$$

which implies by $\left[6\right.$, Theorem 5] the factorization diagram for two measures $\mu_{1}$ and $\mu_{2}$ that are Rybakov measures for $m_{R}$ and $m_{S^{\prime}}$, respectively.

To finish the paper we come back to the consequences of the $(p, q)$ boundedness requirements for the kernel on the $(p, q)$-th power factorability of the corresponding kernel operator. The following result can be considered in sense a variant of Proposition 2.5. It can be applied for instance in the case of the Hille-Tamarkin operators.

Proposition 4.4. Let $X(\mu)$ and $Y(\mu)$ be Fatou and order continuous Banach function spaces such that $Y(\mu)^{\prime}$ is order continuous. Let be $1<p, q<\infty$. Assume that $X(\mu)$ and $Y(\mu)$ are such that $X^{L^{p^{2}}}$ and $\left(Y^{\prime}\right)^{L^{q^{2}}}$ are Banach function spaces. Let $T_{K}: X(\mu) \rightarrow Y(\mu)$ be a kernel operator with kernel $K: \Omega \times \Omega \rightarrow\left[0,+\infty\left[\right.\right.$ such that $K \in L^{1}(\mu \otimes \mu)$ and \|\|$K_{x}\left\|_{p^{\prime}}\right\|_{q^{\prime}}<\infty$, then $T_{K}$ is $(p, q)$-th power factorable. 
Proof. First notice that by Remark 2.3 the fact that $X^{L^{p^{2}}}$ is a Banach function space implies the embedding of $X_{[p]}$ into $L^{p}(\mu)$. The same holds for $Y_{[q]}$ and $L^{q}(\mu)$. Applying this fact and Hölder's inequality twice we obtain

$$
\begin{aligned}
\left|\left\langle T_{K} f, g\right\rangle\right| & =\left|\int_{\Omega} \int_{\Omega} K(x, y) f(y) g(x) d y d x\right| \\
& \leq\|f\|_{p} \int_{\Omega}\left|\left\|K_{x}\right\|_{p^{\prime}}(x)\right||g(x)| d x \\
& \leq\|\| K_{x}\left\|_{p^{\prime}}\right\|_{q^{\prime}}\|g\|_{q}\|f\|_{p} \\
& \leq\|\| K_{x}\left\|_{p^{\prime}}\right\|_{q^{\prime}}\|f\|_{X(\mu)_{[p]}}\|g\|_{Y(\mu)^{\prime}{ }_{[q]}} .
\end{aligned}
$$

\section{References}

1. F. Albiac and N.J. Kalton, Topics in Banach space theory, Graduate Text in Mathematics, vol. 233. Springer, New York, 2006.

2. C. Bennett and R. Sharpley, Interpolation of Operators, Academic Press, Inc., Boston, 1988.

3. J. M. Calabuig, O. Delgado and E. A. Sánchez Pérez, Generalized Perfect Spaces, Indag. Math. 19 (2008) 359-378.

4. J. M. Calabuig, O. Delgado and E. A. Sánchez-Pérez, Factorizing operators on Banach function spaces through spaces of multiplication operators, J. Math. Anal. Appl. 364 (2010) 88-103.

5. J. Diestel and J. J. Uhl, Vector Measures, Math. Surveys, vol. 15, Amer. Math. Soc., Providence, R.I., 1977.

6. A. Fernández, F. Mayoral, F. Naranjo, C. Sáez and E. A. Sánchez-Pérez, Spaces of integrable functions with respect to a vector measure and factorizations through $L^{p}$ and Hilbert spaces, J. Math. Anal. Appl. 330 (2007) 1249-1263.

7. O. Galdames and E.A. Sánchez Pérez, Optimal range theorems for operators with $p$-th power factorable adjoints, Banach J. Math. An. 6 no. 1 (2012) 63-71.

8. J. Lindenstrauss and L. Tzafriri, Classical Banach Spaces II, Springer, Berlin, 1979.

9. S. Okada, W. J. Ricker and E. A. Sánchez Pérez, Optimal Domain and Integral Extension of Operators acting in Function Spaces, Operator Theory: Adv. Appl., vol. 180, Birkhäuser, Basel, 2008.

10. Y. Raynaud and P. Tradacete, Interpolation of Banach lattices and factorization of $p$-convex and q-concave operators, Int. Eq. Oper. Theory, 66 (2010) 79-112.

11. S. Reisner, Operators which factors through convex Banach lattices, Canadian J. Math., 32 (1980) 1482-1500.

12. A.R. Schep, Minkowski's integral inequality for function norms, in Operator Theory in Function Spaces and Banach lattices, 299-308, Operator Theory: Adv. Appl., vol.75, Birkhäuser, Basel, 1995.

13. A. C. Zaanen, Integration, 2nd rev. ed. North Holland, Amsterdam; Interscience, New York, 1967. 
O. Galdames Bravo and E. A. Sánchez Pérez

Instituto Universitario de Matemática Pura y Aplicada, Universidad Politécnica de Valencia Camino de Vera s/n, 46022 Valencia. Spain.

e-mail: orgalbra@posgrado.upv.es, easancpe@mat.upv.es 